\title{
Underwater Object Localization using the Spinning Propeller Noise of Ships Based on the Wittekind Model
}

\author{
Mojgan Mirzaei Hotkani, Seyed Alireza Seyedin, Jean-Francois Bousquet
}

\begin{abstract}
The purpose of this article is to localize underwater objects based on the noise reflection of the propeller rotation in cavitation mode. In the proposed method, the propeller noise, which plays the role of pings in active sonar, is modeled by the Wittekind method. As such, an echo is continuously received by a vertical and uniform linear hydrophone array due to reflection from the underwater targets. The challenges associated with the underwater channels are simulated by the ocean model in COMSOL. Specifically, to model the propagation of underwater acoustic in this channel, the Helmholtz equation is solved using COMSOL. Finally, localization is performed by comparing the Delay \& Sum algorithm and the multiple signal classification (MUSIC) algorithm in MATLAB. According to the simulation results, the proposed method is able to detect the position of the target and the propeller approximately, although the multipath phenomenon causes adverse effects on the results. The narrowband MUSIC algorithm is used in the proposed method at the frequency of the strongest intensity.
\end{abstract}

Keywords: Helmholtz equation, Hydrophone array, localization, MUSIC algorithm, Propeller noise, Wittekind method.

\section{INTRODUCTION}

\section{Commercial vessels are the main source of noise}

production in the ocean and they are an increasing concern because they pollute the marine environment. So, there has been a growing interest to model the underwater radiated noise level of a ship by relating the spectral components of noise to the naval architectural features of the vessels. Several underwater noise modeling tools from vessels have been designed to distinguish noise sources based on measurements made since the Second World War. Historically, the most widely used source model was proposed by Ross [1]. This model was based on the first immense database of ship noise. It was evaluated from the surface vessel noise measurements in the range of America, Canada and Great Britain during the Second World War.

Revised Manuscript Received on February 05, 2020.

* Correspondence Author.

Mojgan Mirzaei Hotkani, Department of Electrical Engineering, Ferdowsi University of Mashhad, Mashhad, Iran. E-mail: m.mirzaei@mail.um.ac.ir.

Seyed Alireza Seyedin*, Department of Electrical Engineering, Ferdowsi University of Mashhad, Mashhad, Iran. E-mail: seyedin@ um.ac.ir.

Jean-Francois Bousquet, Department of Electrical \& Computer Engineering, Dalhousie University, Halifax, Canada. E-mail: JBousquet@Dal.Ca.

(C) The Authors. Published by Blue Eyes Intelligence Engineering and Sciences Publication (BEIESP). This is an open access article under the CC BY-NC-ND license (http://creativecommons.org/licenses/by-nc-nd/4.0/)
Another approach is proposed by Wales and Heitmeyer, which is designed using a statistical analysis from 54 source spectra over the frequency band from $30 \mathrm{~Hz}$ to $1200 \mathrm{~Hz}$ [2].

Recently, the Wittekind noise source model was presented for the Ship Traffic Emission Assessment Model (STEAM) which noise source propagates based on architectural features of vessels [3]. The Wittekind noise source model separates the ship noise into a combination of three portions, which are due to low and high frequency cavitation as well as machinery noise. These noise sources are related to the vessel specifications, such as displacement, hull shape and machinery characteristics. This will be described in Section III.

The focus of the present study is commercial shipping, and when these ships navigate at service speed, cavitation at the propeller is the prevailing noise source, through a large spectral band.

The rotation of the blades around the propeller axis causes the cavitation phenomenon. Specifically, cavitation causes a rapid changes of pressure in a liquid, which leads to small vapor-filled cavity formations in places where the pressure is relatively low. At higher pressures, these cavities, called "bubbles" or "voids", collapse and can generate an intensive shock wave.

The purpose of this paper is to estimate the target location using an innovative passive technique in heterogeneous underwater medium in which the propeller noise plays the role of pings for active sonar. This noise is modeled by the Wittekind method and therefore it acts as a signal that is continuously released from the propeller and is reflected from the underwater targets. The recurring echo is continuously received by the towable hydrophone array. The geographic location of this array is available at any moment. Finally, using existing localization algorithms, the direction of the target can be determined.

The passive localization of an unknown acoustic source is a challenging problem. There has been considerable attention to this issue. Several methods have been developed which work not only in ideal simulations but also in feasible frameworks that include passive localization studies [4-9]. Existing passive localization methods use based on three types of measurements: time delay of arrival (TDOA), direction of arrival (DOA), and the received signal strength or energy. The DOA can be estimated by extracting the phase difference measured at receiving sensors [10]. TDOA is suitable for broadband acoustic source localization and is also applicable for the acoustic source that emits a coherent and narrowband signal. 
It has been extensively investigated in [10]. Also, localization algorithms related to DOA, such as MUSIC, ESPRIT, ROOT MUSIC and MVDR are reviewed in [11-13]. There are other famous localization methods such as matched field processing (MFP) [14], that are not included in these three categories. The MFP algorithm has commonly been used in shallow underwater conditions for source detection and localization using a hydrophones array.

The remainder of this paper is organized as follows. In Section II, the challenges associated to underwater acoustic channels are described. The Wittekind model is defined in Section III and in Section IV, simulation results are presented. Finally, the conclusions are explained in Section V.

\section{CHALLENGES OF UNDERWATER ACOUSTIC CHANNELS}

The underwater acoustic channels are one of the most difficult communication environments. Three main factors describe the acoustic propagation: attenuation that increases with increasing frequency, time-varying multipath propagation, and a depth-dependent speed of sound. One of the outstanding features of the acoustic channels is the fact that the path loss depends on the frequency of the signal. This dependence is the result of absorption. In addition, it also has a spreading loss, which is directly related to distance.

In an underwater acoustic channel, the noise consists of ambient noise and site-specific noise. Ambient noise is always in the background of the deep sea. Site-specific noise, on the contrary, exists only in certain places. For example, ice cracking in polar regions. Ambient noise is often approximated as a colored Gaussian process. In contrast, site-specific noise often contains non-Gaussian effects.

The sound reflections at the surface, bottom and any other targets in the water produce multipath arrivals in the ocean.

Two sources of channel time variability are including intrinsic changes that occur in the propagation medium such as currents and, also transmitter/receiver motion (i.e., Doppler spreading due to the changing path length) [15].

In the underwater medium, the material conditions such as water salinity, temperature, and pressure derive a sound speed profile that is dependent on the depth [16]. Actually, the sound speed profile (SSP) defines the dependence of sound speed as a function of the depth. The change of sound speed causes the acoustic wave bend and spread along a curve.

In this study, the effects of acoustic attenuation, the reflection from the ocean surface, bottom and target and the SSP model for simulation are considered, and other challenges are neglected.

It is often necessary to know how the underwater acoustic propagation behaves by modeling the underwater channel in software. The underwater sound intensity $\psi$ produced in an inhomogeneous medium can be developed to solve the wave equation defined as [21]:

$\nabla^{2} \psi-c^{2} \frac{\partial^{2} \psi}{\partial^{2} t}=f(r, t)$

where $f(r, t)$ represents the volume injection as a function of space $r$ and time $t$. Also, $c$ is the speed of sound that varies with depth. There are various numerical and analytical methods to solve the above equation as described in [17]. The Helmholtz equation indicates a time-independent form of wave propagation. It is yielded by applying the technique of separation of variables to decrease the complexity of the analysis. It is defined as:

$\left[\nabla^{2}+k^{2}(r)\right] \psi(r, \omega)=f(r, \omega)$,

where $k(r)$ is the medium wavenumber.

In this work, the Pressure Acoustics module (Frequency Domain Library) is used in COMSOL to solve (2).

\section{WITTEKIND MODEL}

The Wittekind model depends on [3]:

- The displacement;

- The cavitation inception speed;

- A block coefficient as an indicator of wake field variations; (The block coefficient is the ratio of the displacement to length $\times$ breadth $\times$ draft of the ship).

- The mass of diesel engine(s); and

- A Boolean flag indicating resilient mounting of the diesel engine.

These parameters are combined to the three main factors of underwater propagated noise: low-frequency cavitation noise, denoted by $F_{1}$, high-frequency cavitation noise denoted by $F_{2}$, and diesel engine noise denoted by $F_{3}$. Each of these factors should be considered as an averaged sound pressure level in one-third octave bands. The overall source level (SL) yield by the following equation:

$S L=10 \log \left(10 \frac{F_{1}}{10}+10 \frac{F_{2}}{10}+10 \frac{F_{3}}{10}\right)$.

In this paper, a standard selection of center frequencies with one at $10 \mathrm{~Hz}$ is considered, then the rest of $f$ can be yielded by the $1 / 3$-octave ratio.

The equation for $F_{1}$ is obtained by a curve fitting to data from Arveson and Vendittis [17], and leads to:

$F_{1}=\sum_{n=0}^{5} c_{n} f^{n}+A\left(V, V_{c}, c_{B}\right)+B\left(D_{T}\right)$,

where $V_{c}$ is the cavitation-inception speed, $c_{B}$ is the ship hull block coefficient, and the coefficient values $c_{i}$ are given in Table I. $A\left(V, V_{c}, c_{B}\right), B\left(D_{T}\right)$ in (4) model the scaling with speed and displacement respectively and are defined by:

$A=80 \log _{10}\left(\frac{4 c_{B} V}{V_{c}}\right), \quad B=\frac{20}{3} \log _{10} \frac{D_{T}}{D_{T, \text { ref }}}$,

where $D_{T \text {, ref }}=10000 \mathrm{t}$, is a reference displacement. Also, $F_{1}$ is suitable for low frequencies below $400 \mathrm{~Hz}$.

For the high-frequency cavitation noise, $F_{2}$, again a curve fitting is used based on databases [3], which yields:

$F_{2}=-5 \ln f-\frac{1000}{f}+10+B\left(D_{T}\right)+C\left(V, V_{c}, c_{B}\right)$.

The function $C$ models the effect of speed. It is defined in (7).

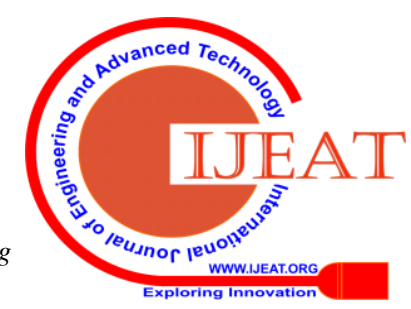


Table- I: Coefficients in the expression for $F_{1}$.

\begin{tabular}{|c|c|c|c|c|c|c|} 
Coefficient & \multicolumn{1}{c}{$\boldsymbol{c}_{\mathbf{0}}$} & \multicolumn{1}{c}{$\boldsymbol{c}_{\mathbf{1}}$} & $\boldsymbol{c}_{2}$ & $\boldsymbol{c}_{3}$ & $\boldsymbol{c}_{4}$ & $\boldsymbol{c}_{5}$ \\
\hline Value & 125 & 0.35 & $\begin{array}{c}-8 \times \\
10^{-3}\end{array}$ & $\begin{array}{c}6 \\
\times 10^{-5}\end{array}$ & $\begin{array}{c}-2 \times \\
10^{-7}\end{array}$ & $\begin{array}{c}2.2 \times \\
10^{-10}\end{array}$ \\
\hline
\end{tabular}

$C\left(V, V_{c}, c_{B}\right)=60 \log _{10}\left(\frac{1000 c_{B} V}{V_{c}}\right)$.

Finally, for the engine noise $F_{3}$, the following equation is defined.

$F_{3}=10^{-7} f^{2}-0.01 f+140+D(m, n)+E$,

where $D$ is a factor modeling the engine mass and the number of engines. Also, $E=0$ indicates that the engine is resiliently mounted, and $E=15$ indicates that the engine is rigidly mounted. Finally,

$D(m, n)=15 \times \log (m)+20 \times \log (n)$,

where, $m$ is the engine mass in tons, and $n$ is equal to the number of engines operating at the same time.

\section{SIMULATION RESULTS}

The main purpose of this article is to localize the underwater targets based on the reflection of the noise emitted by the rotation of the commercial vessel propeller. This noise often occurs in cavitation mode which propagates through the channel. Therefore, the noise that plays the role of a ping in active sonars is received by a vertical and uniform hydrophone array after encountering underwater targets on which they are reflected. The following steps describe these procedures briefly.

1-Simulating the noise of the ship using the Wittekind model.

Considering the obtained signal of the Wittekind model as the sound source signal in COMSOL, and simulating the acoustics propagation in COMSOL using the Helmholtz equation and ocean model in the presence of a target.

2- Extracting the signals of hydrophones from COMSOL, and localizing the sound source (propeller of the ship) and target using MUSIC and Delay \& Sum algorithms.

The Wittekind model (3) with the following parameters is used to simulate the noise which emitted by the rotation of the commercial ship's propeller in cavitation mode [19]:

- Ship speed=14 knots.

- Displacement=33 000 tons.

- $c_{B}=0.78$.

- $V_{C}=9 \mathrm{kn}$.

- Two diesel engines of 30 tons.

- The engine mounting parameter, $E=0$.

Fig. 1 shows the simulation results of the Wittekind model for the commercial ship with the above parameters. The signal propagation in sea water follows the Helmholtz equation, so the source level of this ship noise was injected into a sound propagation model based on the approximation of the Helmholtz equation. Specifically, the Pressure Acoustics module (Frequency Domain) was used in COMSOL for solving the Helmholtz equation.

The Acoustics Module includes many specialized formulations and material models that can be used for

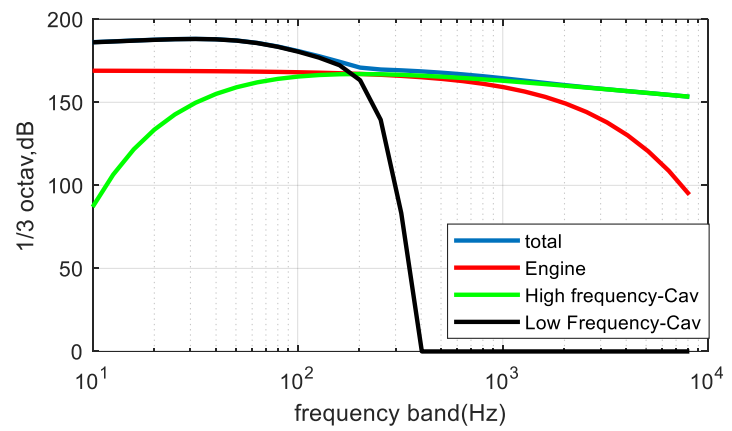

Fig. 1. Modeled source level of an example ship.

dedicated application areas. This module supports time-harmonic (frequency domain), Eigen frequency, modal, and transient studies for all fluids (depending on the acoustic equations solved). In this paper, all the conditions of the real ocean environment are considered in Section IV.A: specifically, Section IVA through Section IV.E, detail respectively a) the problem geometry, b) the simulation model, c) summarizes the localization test.

\section{A. Description of the physical environment}

The ocean geometry is considered as a 3D cube with a dimension of $1000 \mathrm{~m}$ in length, $100 \mathrm{~m}$ in width and $500 \mathrm{~m}$ in depth. The coordinates are in (length, width, and depth), and the reference point is at the surface, at the location of the propeller. Ocean water features are defined according to Table II.

Table- II: Ocean Water Features.

\begin{tabular}{c|c}
\hline Features & Value \\
\hline Temperature & $293.15 \mathrm{~K}$ \\
\hline Salinity & $0.2 \mathrm{~g} / \mathrm{kg}$ \\
\hline $\mathrm{pH}$ & 8 \\
\hline Sound speed in water & According to sound speed profile \\
\hline Water density & $1000 \mathrm{~kg} / \mathrm{m}^{3}$ \\
\hline
\end{tabular}

The Francois and Garrison Equations have been used to model the sound attenuation in the ocean environment [22]. Also, the sound speed in the water follows the sound speed profile according to Fig. 2.

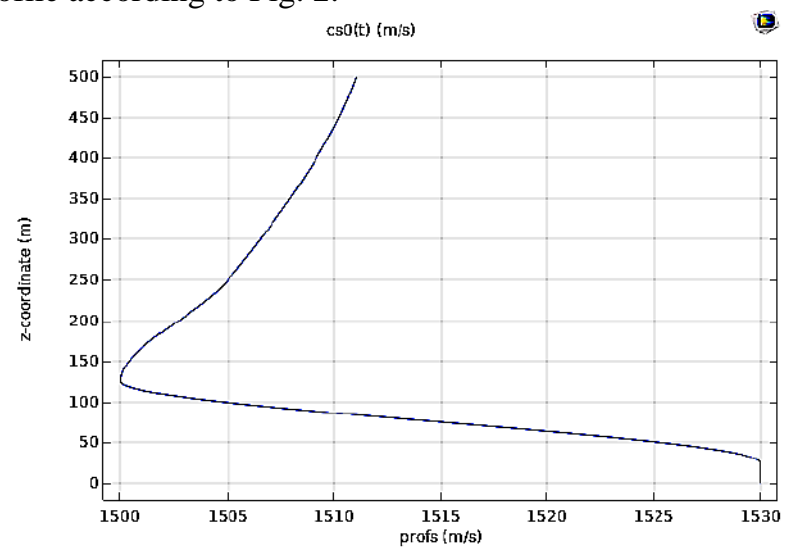

Fig. 2. Profile of sound speed.

Above the ocean surface, the volume is filled by a three-dimensional cube of air with a sound speed of $350 \mathrm{~m} / \mathrm{s}$, a viscosity of $1 \mathrm{~Pa} / \mathrm{s}$ with dimensions $1000 \mathrm{~m}$ in length, $100 \mathrm{~m}$ in width and $10 \mathrm{~m}$ in depth. 
A target (a hollow sphere) is considered with a radius of 3 meters deep below the sea surface, with the coordinates equal to $(200 \mathrm{~m}, 50 \mathrm{~m}, 100 \mathrm{~m})$.

In addition to the above, the modeled source level by the Wittekind model is considered as a point noise source at coordinates (5 m, $50 \mathrm{~m}, 480 \mathrm{~m}$ ).

\section{B. Simulation Model}

In this section, how the physics are modelled is presented. Using COMSOL, the approximation of the Helmholtz equation is simulated and initialized the parameters of the problem with the boundary conditions. Then, the total geometry of the problem is meshed. To increase the processing speed, the ocean and air environments were meshed with coarse resolution. Also, the target was meshed with fine resolution. Fig. 3 shows an overview of the meshed geometry of the problem in the 3-dimensional model using COMSOL. Finally, after implementing and running this problem, the pressure diagrams in terms of frequency, ocean dimensions, and wave front of the modeled source level of an example ship is extracted. The diagrams are shown in Figs 4-5.

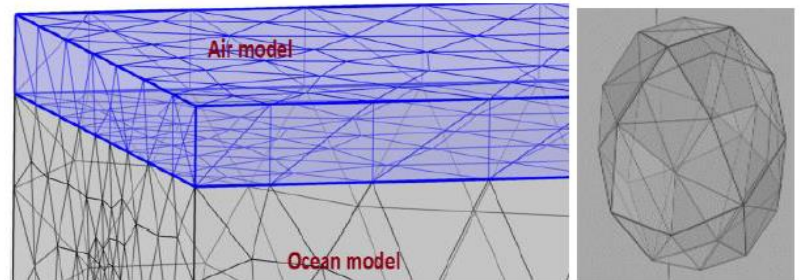

Fig. 3. An overview of the meshed geometry of the problem.

Fig. 4. shows the underwater acoustic propagation from a modeled source level of propeller noise solved by the Helmholtz equation in $\mathrm{db}$. There is the strongest acoustic pressure level around the propeller position. To provide better clarity used the wave front diagram in Fig. 5.

The propagation of sound wave is shown in the red areas. The effects of reflecting waves are considered from the surface and floor of the ocean.

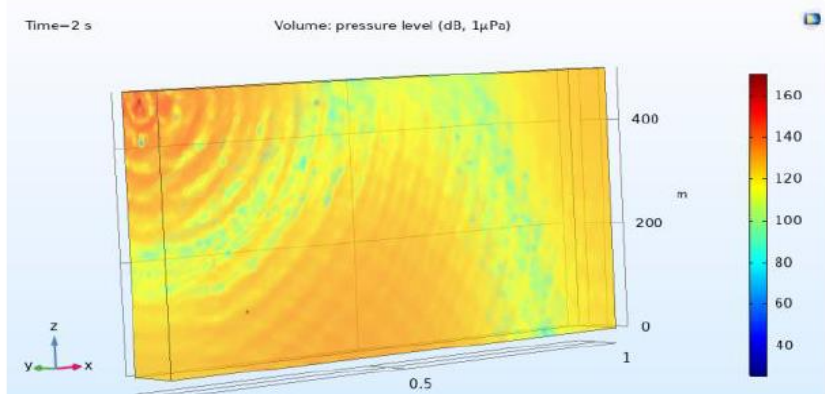

Fig. 4. The underwater acoustic propagation using a modeled source level of propeller noise.

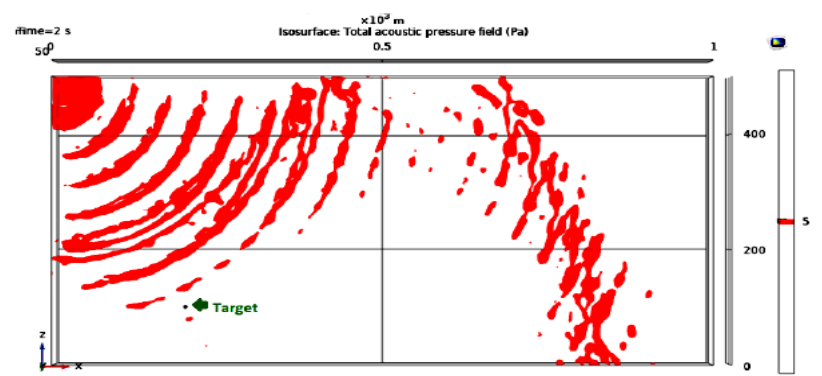

Fig. 5. The wave front of acoustic pressure of the modeled source of propeller noise.

\section{Localization test}

In this section, a uniform and vertical linear array of four hydrophones is positioned with a distance of $1 \mathrm{~m}$ from each other. To prevent aliasing, the distance must satisfy:

$d \leq \frac{\lambda_{\min }}{2}$,

where $d$ is the distance between the two hydrophones and $\lambda_{\min }$ is the minimum value of wavelength.

The coordinates of hydrophones are given in TABLE III.

The target position was estimated approximately based on the data obtained from the hydrophone array and using beamforming algorithms such as MUSIC [11] and Delay \& Sum [20].

\begin{tabular}{|c|c|c|c|}
\hline & Location (Z) & Location $(\mathbf{Y})$ & Location $(\mathrm{X})$ \\
\hline Hydrophone \#1 & $301 \mathrm{~m}$ & $50 \mathrm{~m}$ & $300 \mathrm{~m}$ \\
\hline Hydrophone \#2 & $300 \mathrm{~m}$ & $50 \mathrm{~m}$ & $300 \mathrm{~m}$ \\
\hline Hydrophone \#3 & $299 \mathrm{~m}$ & $50 \mathrm{~m}$ & $300 \mathrm{~m}$ \\
\hline Hydrophone \#4 & $298 \mathrm{~m}$ & $50 \mathrm{~m}$ & $300 \mathrm{~m}$ \\
\hline
\end{tabular}

The signal from the first hydrophone in the array is shown in Fig. 6. The propeller noise signal generated by underwater vessels is a cyclostationary process, so using signal spectrogram processing, the shaft frequency of the propeller can be identified. Based on the spectrogram of the signal, the maximum frequency is at $31.25 \mathrm{~Hz}$. This may be due to the rotation frequency of the shaft according to Fig. 7. Also, this frequency was considered to find the direction of arrival using the MUSIC algorithm.

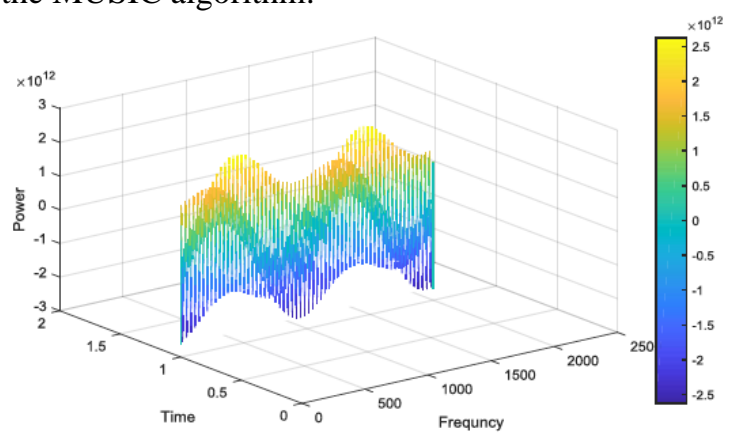

Fig. 6. Time - Frequency and power chart of the first hydrophone signal received.
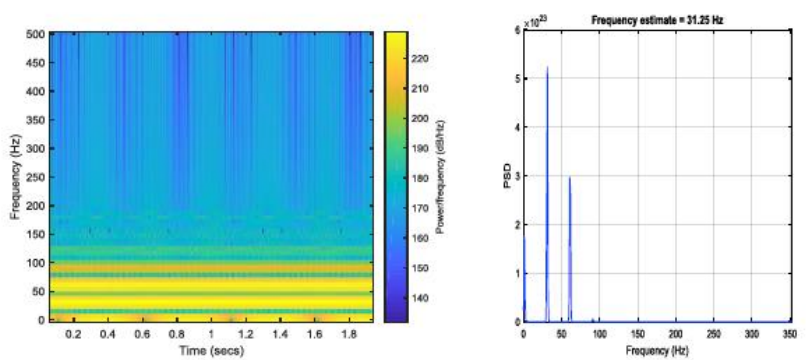

Fig. 7. Spectogram of the first hydrophone signal received.

First, an analysis is performed in the frequency domain in COMSOL, then transferred to the time domain using the IFFT. Finally, using two localization algorithms (MUSIC and

Delay \& Sum) based on DOA, the direction of the target and the ship are determined. 
According to the geometry of this problem, the target and ship are located at angles of 153.4 degrees and 58.5 degrees relative to the length of the array, respectively. Fig. 8 and Fig. 9 are the results of the delay and sum beamforming. Based on the energy intensity shown in Fig. 8, Zones A and B are the estimated positions of the ship and the target. Other areas are caused by echoes of the ocean floor and surface. As shown, the signal energy is reduced at high depths. Although the delay and sum method was not able to estimate the exact positions with high accuracy (based on the location of the target in section $\mathrm{C}$ ), we conclude that the proposed idea, which is a form of opportunistic use of ambient noise, can provide coarse estimation at low cost and it will also be a new subject in various fields in oceanography.

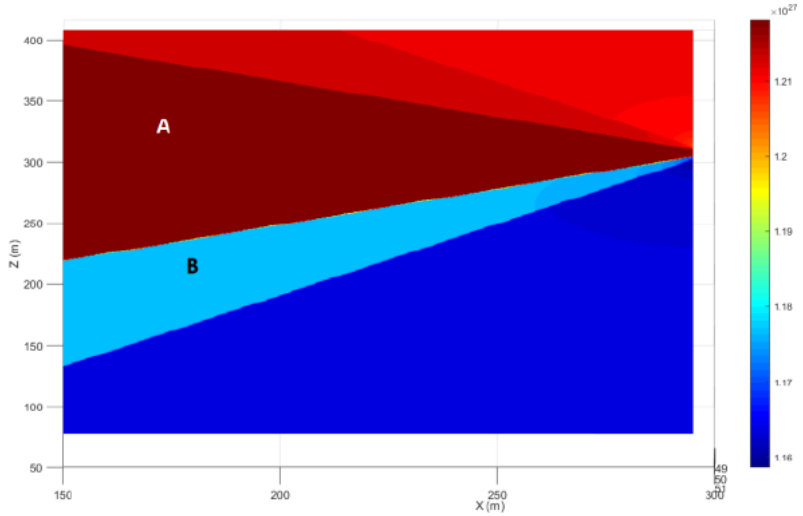

Fig. 8. Localizing results by the Sum and Delay algorithm in terms of distance.

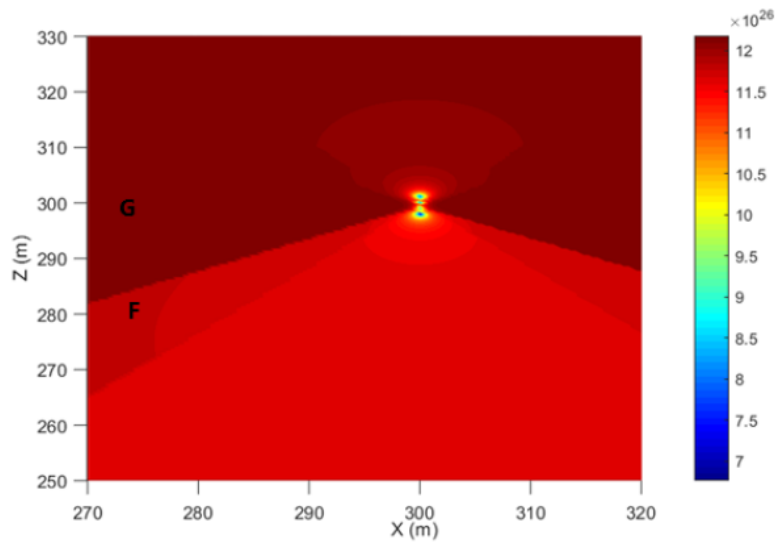

Fig. 9. Another view from Fig. 8.

The bright spots in Fig. 9 represent the array hydrophones. Also, the $G$ and $F$ zones are the estimated positions of the ship and the target. Other areas are caused by echoes of the ocean floor and surface.

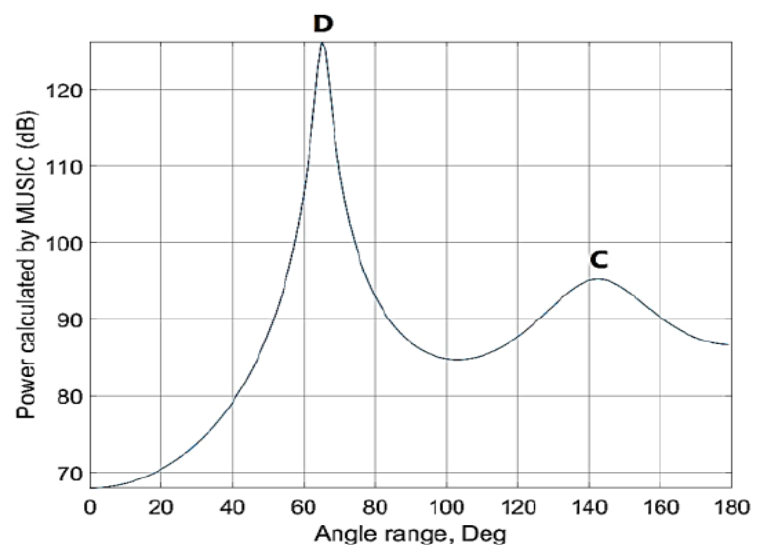

Fig. 10. DOA estimation of target and ship signals.
Figure 10 shows the localization result of the MUSIC algorithm with peaks at $\mathrm{D}=65^{\circ}$ and $\mathrm{C}=143^{\circ}$, that represent the estimated direction of the ship and the target respectively. As shown, the MUSIC algorithm has better performance than the Sum and Delay with less error difference and side lobes in the beam pattern.

\section{CONCLUSION}

In this paper, the use of the noise generated by the spinning propeller in cavitation mode to detect the positions of the underwater targets was proposed. For modeling the propeller noise, the Wittekind model was used based on the parameters of commercial vessel propeller. This noise is a form of signaling that can play the role of pings in active sonars. The recurrent echo was continuously received by a vertical and uniform linear hydrophone array due to reflection from the underwater targets. The signal generated by the Wittekind model was used as the source signal in COMSOL. COMSOL simulated the Helmholtz equation in the ocean environment. Then, the signals of hydrophones were extracted from COMSOL. Finally, using well-known localization algorithms, such as the Delay-Sum, and MUSIC, the direction of the target was determined. According to the simulation results, the proposed method by the MUSIC algorithm was well able to detect the position of the target and the propeller approximately compared to the delay-sum method, due to the multipath phenomenon in the ocean. So, the proposed idea, which is a form of opportunistic use of ambient noise, will be practical and it will also receive significant attention in various fields in oceanography.

\section{REFERENCES}

1. D. Ross. Mechanics of underwater noise. Peninsula Publishing, 1976.

2. S. C. Wales and R. M. Heitmeyer. "An ensemble source spectra mode for merchant ships". Journal of the Acoustical Society of America, 111:1211-1231, 2002.

3. D. K. Wittekind. A simple model for the underwater noise source level of ships. Journal of Ship Production and Design, 30(1):1-8, 2014.

4. S. Poursheikhali and H. Zamiri-Jafarian, "Received signal strength-based localization in inhomogeneous underwater medium,' Signal Processing, 2019.

5. J. Li, Q. H. Lin, K. Wang, and C. Y. Kang, "Performance Analysis for Focused Beamformers in Passive Underwater Acoustic Localization," IEEE Access, 2018.

6. B. Marxim Rahula Bharathi and A. R. Mohanty, "Underwater Sound Source Localization by EMD-Based Maximum Likelihood Method,' Acoust. Aust., 2018.

7. D. Zhao, W. Seong, K. Lee, and Z. Huang, "Shallow water source localization using a mobile short horizontal array," J. Syst. Eng. Electron., 2013.

8. P. M. Ameer and L. Jacob, "Localization Using Ray Tracing for Underwater Acoustic Sensor Networks," IEEE Commun. Lett., vol. 14, no. 10 , Oct. 2010, pp. 930-932.

9. H. Ramezani , H. Jamali-Rad , G. Leus , "Target localization and tracking for an iso- gradient sound speed profile, "IEEE Trans. Signal Process. 61, 2013, pp. 1434-1446.

10. J. Benesty, J. Chen, and Y. Huang, Microphone array signal processing. Springer, 2008.

11. H. Tang, "DOA estimation based on MUSIC algorithm," thesis Basic level (degree of Bachelor), Linnaeus University, Department of Physics and Electrical Engineering, 2014.

12. C. Qian, L. Huang, and H. C. So, "Improved unitary root-MUSIC for DOA estimation based on pseudo-noise resampling," IEEE Signal Process. Lett., 2014.

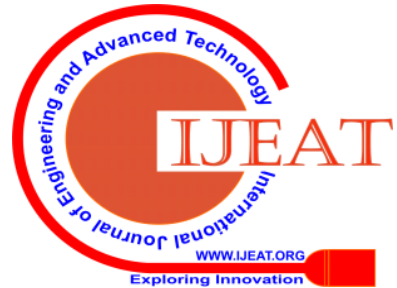


13. W. Suleiman, M. Pesavento, and A. M. Zoubir, "Performance Analysis of the Decentralized Eigendecomposition and ESPRIT Algorithm," IEEE Trans. Signal Process., 2016.

14. P. N. Tran and K. D. Trinh, "Adaptive Matched Field Processing for Source Localization Using Improved Diagonal Loading Algorithm," Acoust. Aust., 2017.

15. M. Stojanovic and J. Preisig, "Underwater Acoustic Communication Channels: Propagation Models and Statistical Characterization," IEEE Commun. Mag., 2009.

16. F. B. Jensen et al., "Computational ocean acoustics," in American institute of physics, Springer, New York, 2011, pp. 1-232. DOI: 10.1002/qj.49712152515.

17. P. T. Arveson and D. J. Vendittis. "Radiated noise characteristics of a modern cargo ship". J. Acoust. Soc. Am., 107(1):118-129, 2000.

18. R. J. Urick, Principles of underwater sound. McGraw-Hill, 1983.

19. BONUS SHEBA-project website: https://www.sheba-project.eu/.

20. http://www.labbookpages.co.uk/audio/beamforming/delaySum.html.

21. M. Gorji, H. Ghassemi, and J. Mohamadi, "Calculation of sound pressure level of marine propeller in low frequency." SAGE journal, 2018.

22. R. E. Francois and G. R. Garrison, "Sound absorption based on ocean measurements. Part II: Boric acid contribution and equation for total absorption," J. Acoust. Soc. Am., 1982.

\section{AUTHORS PROFILE}

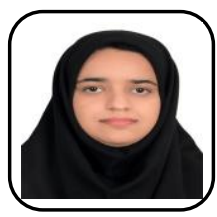

Mojgan Mirzaei Hotkani received the B.S. and the M.S. degrees in Electrical Engineering from Shahid Bahonar University of Kerman, Kerman. Iran. Currently, she is a Ph.D. Candidate at Ferdowsi University of Mashhad, Mashhad, Iran as well as visiting research student at Dalhousie University, Halifax, Canada.

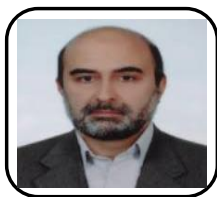

Seyed Alireza Seyedin received the B.S. degree in Electronics Engineering from Isfahan University of Technology, Isfahan, Iran in 1986, and the M.E. degree in Control and Guidance Engineering from Roorkee University, Roorkee, India in 1992, and the Ph.D. degree from the University of New South Wales, Sydney, Australia in 1996. Currently, He is an Associate Professor at Department of Electrical Engineering, Ferdowsi University of Mashhad, Mashhad, Iran.

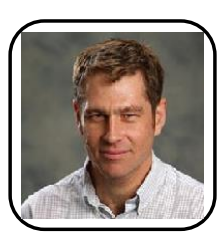

Jean-Francois Bousquet joined the Electrical and Computer Engineering Department at Dalhousie in July 2013. He is a graduate of École Polytechnique de Montréal where he completed his B. Eng. in Electrical Engineering in 2001. He also completed his M.Sc. and Ph.D. degree in Electrical Engineering at the University of Calgary in 2007 and 2011 respectively. Since he joined Dalhousie, he has applied his broad knowledge in field of electrical and computer engineering towards underwater communications to enable underwater networks, using low-power electronics. 